Supporting Information:

\title{
Sequencing orbitides by acid-mediated ring cleavage followed by tandem mass spectrometry
}

Mark F. Fisher ${ }^{*}{ }^{\dagger}$ and Joshua S. Mylne ${ }^{\dagger}$

*Corresponding author: Mark F. Fisher, E-mail: research@markfisher.id.au

+ The University of Western Australia, School of Molecular Sciences \& ARC Centre of

Excellence in Plant Energy Biology (M310), 35 Stirling Highway, Crawley WA 6009, Australia

Figure S1. Total ion current chromatograms and mass spectra showing time series of hydrolysis of cyclo-FVGGTSFD (PLP-12).

Figure S2. Total ion current chromatogram and mass spectrum showing hydrolysis of cycloGLLGITD (PLP-10).

Figure S3. Total ion current chromatogram and mass spectrum showing hydrolysis of cycloGSPLFD (PLP-4).

Figure S4. Total ion current chromatogram and mass spectrum showing hydrolysis of cycloFVGGTSFD (PLP-12). 

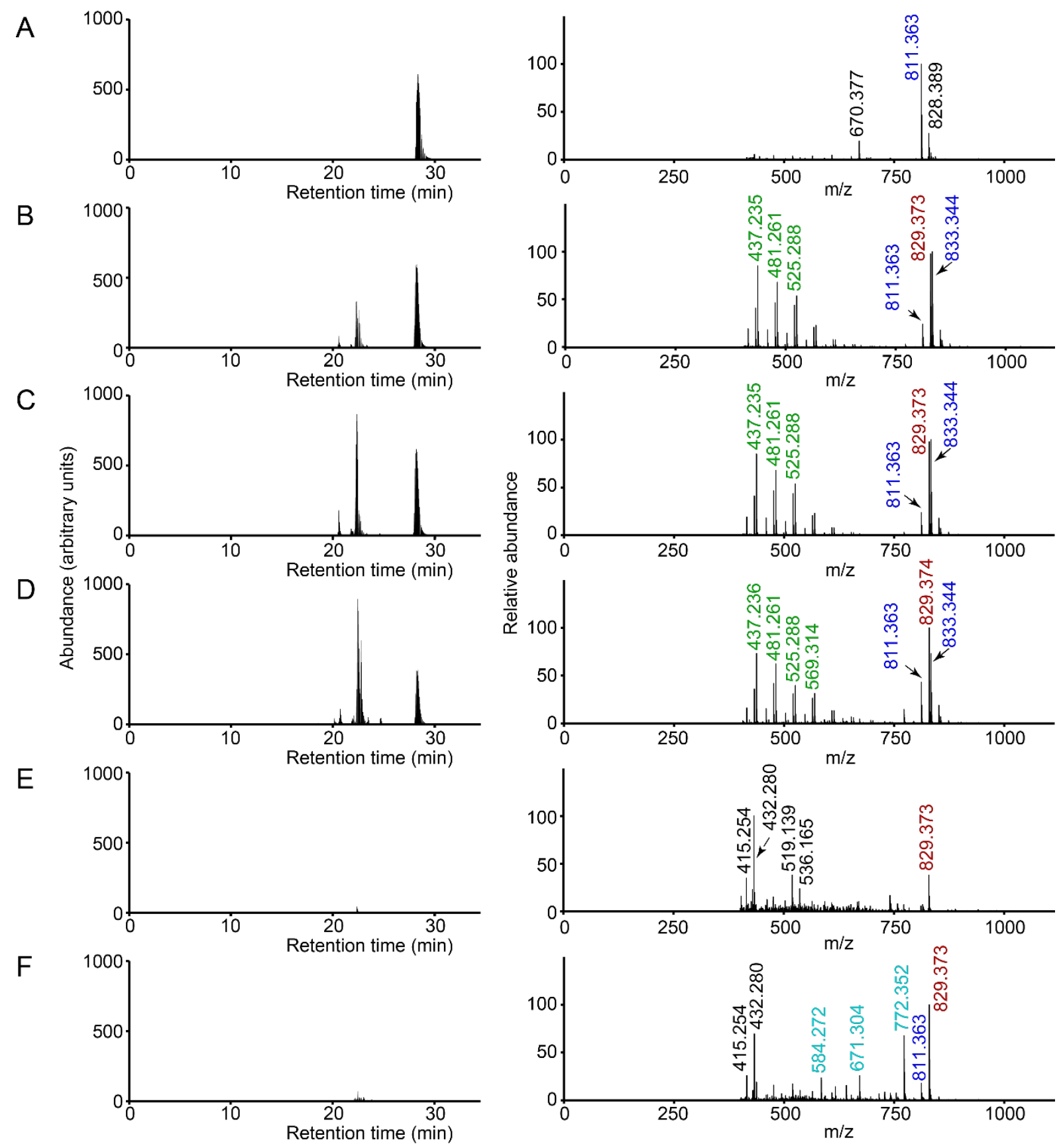

Figure S1. Total ion current chromatograms and mass spectra showing time series of hydrolysis of cyclo-FVGGTSFD (PLP-12). A-F: hydrolysis reactions at $0 \mathrm{~min}$ (control), $10 \mathrm{~min}, 20$ $\mathrm{min}, 30 \mathrm{~min}, 40 \mathrm{~min}$ and 50 minute respectively. Left: total ion current chromatogram for each reaction. Right: mass spectra taken across the whole of each LC-MS experiment, retention times $0-60 \mathrm{~min} . \mathrm{m} / \mathrm{z}$ of cyclic peptide (including sodium adduct) shown in blue, linear hydrolyzed peptide in red, further hydrolysis products in aqua, and putative polyethylene glycol products released from the reaction tubes in green. Uncharacterized peaks (labelled in black) probably represent impurities or further degradation products. 


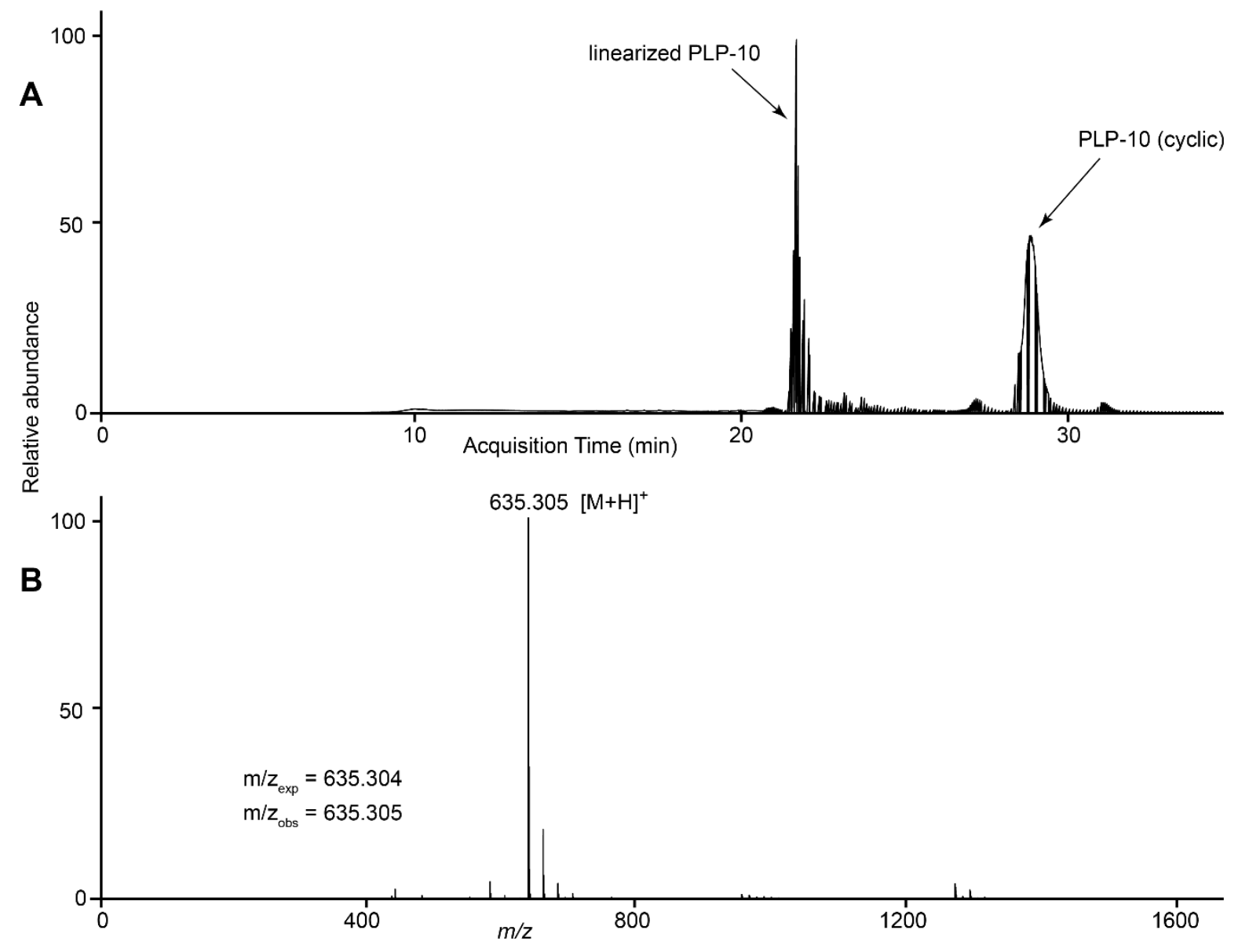

Figure S2. Total ion current chromatogram and mass spectrum showing hydrolysis of cycloGLLGITD (PLP-10). .A: the TIC shows peaks corresponding to the original cyclic peptide and the hydrolyzed linear form. B: The mass spectrum at the retention time of linearized PLP-10 showing that the observed $\mathrm{m} / \mathrm{z}\left(\mathrm{m} / \mathrm{z}_{\text {obs }}\right)$ closely matches the expected $\mathrm{m} / \mathrm{z}\left(\mathrm{m} / \mathrm{z}_{\text {exp }}\right)$. 


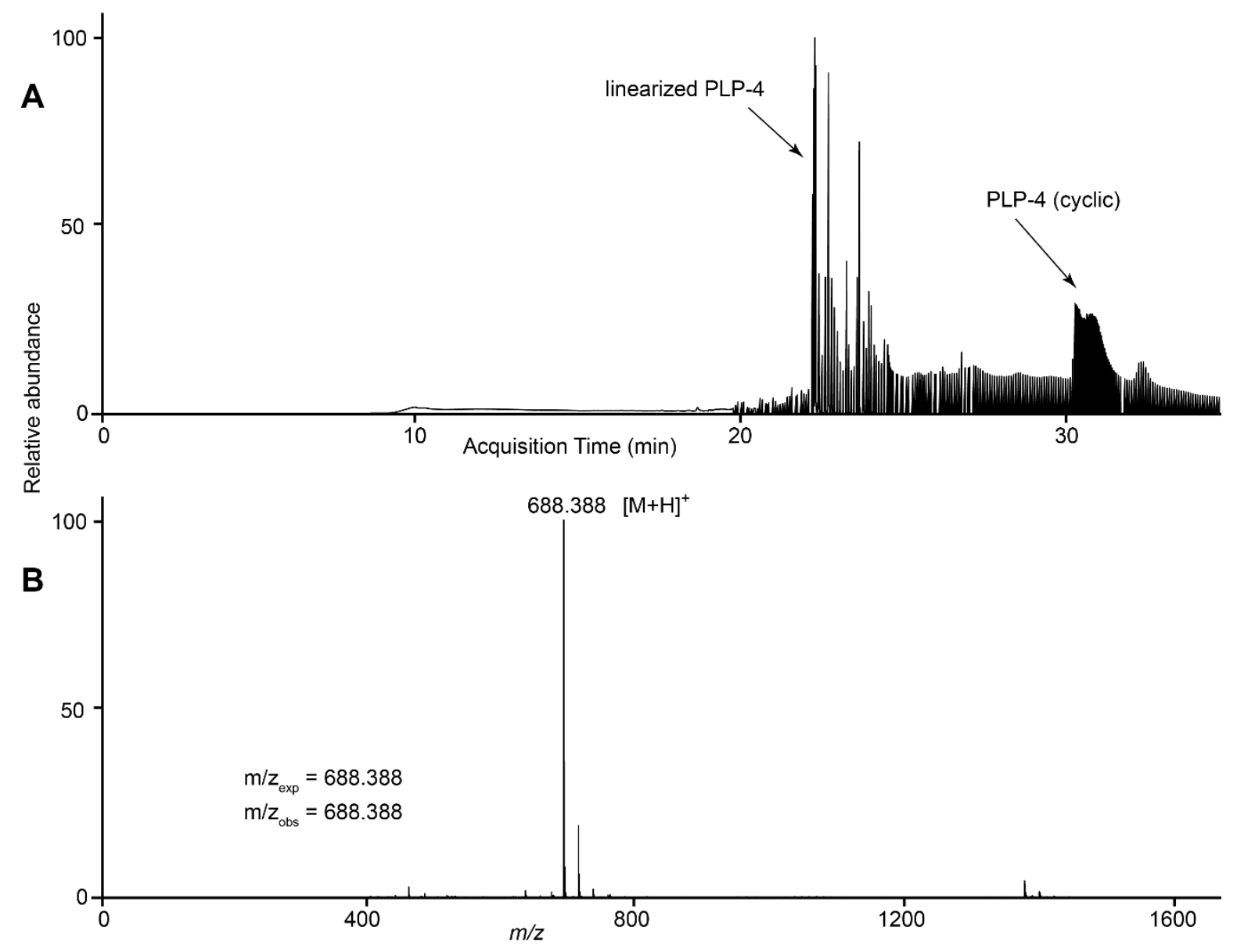

Figure S3. Total ion current chromatogram and mass spectrum showing hydrolysis of cyclo-GSPLFD (PLP-4). .A: the TIC shows peaks corresponding to the original cyclic peptide and the hydrolyzed linear form. B: The mass spectrum at the retention time of linearized PLP-4 showing that the observed $\mathrm{m} / \mathrm{z}\left(\mathrm{m} / \mathrm{z}_{\text {obs }}\right)$ closely matches the expected $\mathrm{m} / \mathrm{z}\left(\mathrm{m} / \mathrm{z}_{\exp }\right)$. 


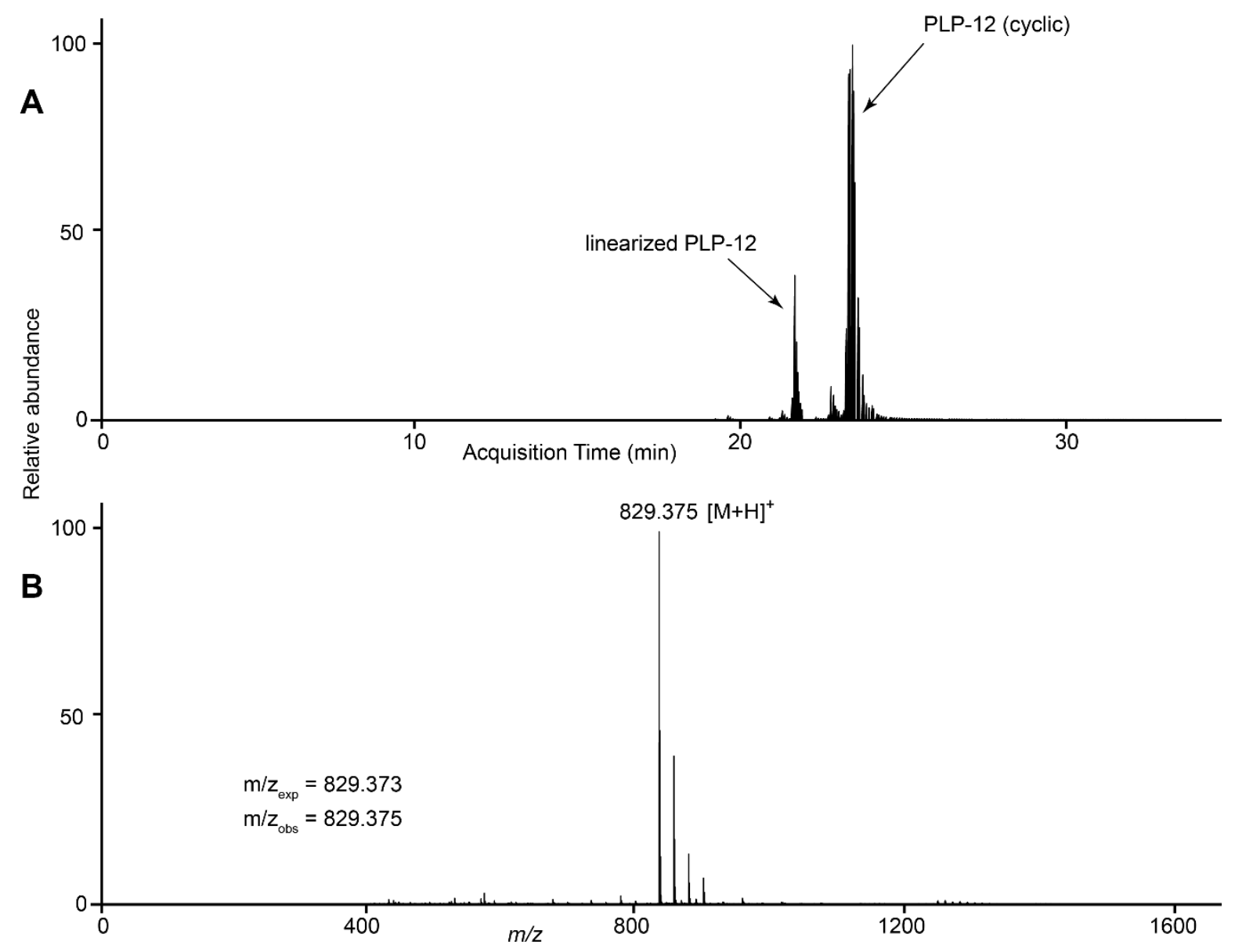

Figure S4. Total ion current chromatogram and mass spectrum showing hydrolysis of cycloFVGGTSFD (PLP-12). .A: the TIC shows peaks corresponding to the original cyclic peptide and the hydrolyzed linear form. B: The mass spectrum at the retention time of linearized PLP-12 showing that the observed $m / z\left(m / z_{\text {obs }}\right)$ closely matches the expected $m / z\left(m / z_{\text {exp }}\right)$. 